\title{
Role of Antioxidants in Female Fertility
}

\author{
Amal AL Mulla ${ }^{*}$, Atif B. E. Fazari ${ }^{2,3}$, Mohamed Elkhouly ${ }^{4}$, Natalie Moghaddam ${ }^{4}$ \\ ${ }^{1}$ Dubai Fertility \& Gynecology Center, Dubai, UAE \\ ${ }^{2}$ Latifa Hospital, Dubai Health Authority, Dubai, UAE \\ ${ }^{3}$ University of Medical Sciences \& Technology, Faculty of Medicine, Khartoum, Sudan \\ ${ }^{4}$ Bourn Hall Fertility Centre, Dubai, UAE \\ Email: *amalalmulla2020@outlook.com
}

How to cite this paper: AL Mulla, A., Fazari, A.B.E., Elkhouly, M. and Moghaddam, N. (2018) Role of Antioxidants in Female Fertility. Open Journal of Obstetrics and Gynecology, 8, 85-91.

https://doi.org/10.4236/ojog.2018.82011

Received: December 18, 2017

Accepted: February 4, 2018

Published: February 7, 2018

Copyright $\odot 2018$ by authors and Scientific Research Publishing Inc. This work is licensed under the Creative Commons Attribution International License (CC BY 4.0).

http://creativecommons.org/licenses/by/4.0/

\begin{abstract}
The last decade has seen a noticeable advance in diagnosing and treating infertility, however, the overall incidence has remained the same, with an estimated $40 \%-50 \%$ resulting from factors related to the female [1]. Unexplained infertility where the cause remains elusive, still accounts for up to $30 \%$ of cases. Increasing evidence demonstrates the significant of antioxidants in all physiological and biochemical processes in humans, including human reproduction.
\end{abstract}

\section{Keywords}

Female Fertility, Antioxidants, Free Radicals, Reactive Oxygen Species, Assisted Reproductive Technique

\section{Introduction}

Free radicals are naturally occurring molecules that result from various biological processes secondary to oxygen metabolism; in addition, they can result from environmental factors including, pollutions, radiations, stress, etc. Accumulation of those free radicals activates a series of reactions that can ultimately lead to cellular damage and death.

Antioxidants systems exist naturally to limit the harmful effects of free radicals, by inactivating them and repairing damaged cells.

This delicate and complicated balance between reactive oxygen species (ROS), and antioxidants is fundamental for proper cell functions [2].

Disturbance of this equilibrium towards the over production of ROS leads to oxidative stress [3] which can result in significant harm to the body, including the female reproduction functions, and biological reactions involved in the complicated process of oocyte maturation and steroidogenesis. 
Considering this fact could go some way in explaining the remaining questions faced in the treatment of human infertility.

\section{Free Radicals}

Free radicals are molecules that are produced naturally in the human mitochondria secondary to oxygen metabolism. Characterized by its ability to produce a series of chemical reactions aiming to regain their stability, due to lacking an electron in their outer shell, such deleterious interaction can alter cell function that ultimately ends with cell death.

Exogenous sources are well recognized, which have similar harmful effects. Two types of free radicals are identified, reactive oxygen species (ROS) and reactive nitrogen species (RNS).

Free radicals produce their effect through lipid oxidation, which is considered the key step in initiating several diseases. Lipid oxidation leads to the accumulation of lipid byproducts, which, due to their toxicity affect the cell membrane permeability and electrophysiological signaling.

The reactions produced are progressive and once started spreads rapidly. Thus, the initial oxidation of only a few lipid molecules can result in significant tissue damage [4].

Presence of efficient antioxidant system in the body are mandatory for continuous deactivation of free radicals.

\section{Antioxidants}

Antioxidants are low molecular weight molecules that can attack free radicals and neutralize them through donating an electron thus reducing their capacity to damage [3]. Such interaction can safely put an end to a series of damaging reactions that can affect vital molecules.

Interestingly the human body is designed to create its own antioxidants and several antioxidant enzymes are recognized.

Glutathione is considered the chief natural antioxidant, as it is very effective in the detoxifying mechanism and maintaining the cell redox state by maintaining the crucial balance between ROS and antioxidants. In addition, its presence is critical for oocyte maturation.

Other antioxidant system components are also important and deficiency of any of the components or altered concentrations among the components may impair the function of the whole system.

Anti-oxidants exert their fundamental role, through four levels of defense, starting by hindering free radicals' formation, and a scavenging mechanism to conquer the propagation reactions, vitamin $\mathrm{E}$ is very potent in such mechanism.

DNA repair systems form a strong defense against oxidative damage. Adaptation is the fourth level of defense where the signal for the production and reactions of free radicals prompts creation and transport of specific antioxidant to the right site [5] (Figure 1). 


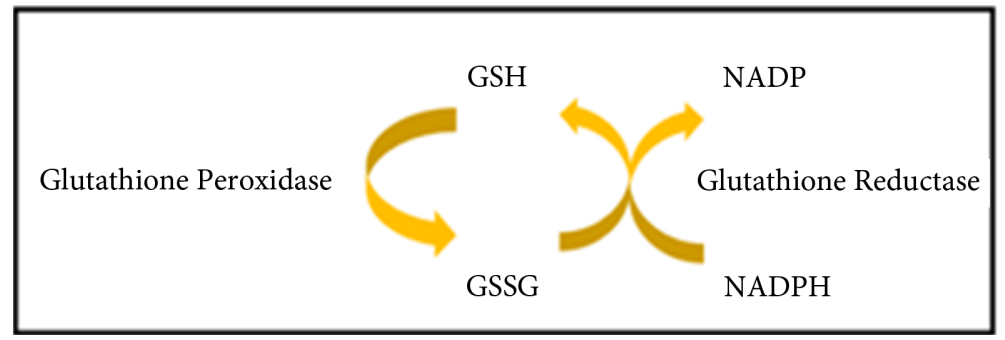

Figure 1. The glutathione system.

Defiency of dietary intake of non-enzymatic antioxidants, has a negative impact on the integrity of the antioxidant system.

\section{Role of Antioxidants in Female Fertility}

Female genital tract function can be disrupted if the antioxidant system has exhausted due to over production of ROS. This can alter oocyte maturation, steroidogenesis, ovulation, in addition it can accelerate granulosa cells apoptosis, which is a naturally occurring phenomenon, involving programmed cell death.

In Vitro experiments have clearly defined, that deficiency of ovarian glutathione accelerates antral follicles atresia, which reflects the high sensitivity of antral follicles to Oxidative Stress. The same applied for the process of fertilization and embryonic development.

Studies have found higher ROS values in women with unexplained infertility, when compared with their fertile counterpart [6].

The natural accumulation of Free radicals with age can very well explain the poorer quality of oocytes encountered in females of advanced age [7].

Psychological stress can lead to accumulation of ROS through adopting unhealthy lifestyle behaviors such as cigarette smoking [8]. ROS resulting from psychological stress will further disturb the granulosa cell function subsquently the reduction of estradiol levels will further reduce the quality and number of retrieved oocytes.

\section{Oxidative Stress and Folliculogenesis}

The ovary is a germ cell reservoir that reflects the female fertility potential. The process of oocyte maturation is one of the most sophisticated processes in the human body involving plethora of complicated biochemical reactions that take place simultaneously and yet not fully understood.

The delicate balance and concentration of ROS have entirely different effects on the oocytes maturation while the physiological level of ROS of (60 - $80 \mathrm{ng}$ / oocyte) may prompt meiotic division completion, increased level of $>80 \mathrm{ng} / \mathrm{oo}-$ cyte can be hazardous [9].

Evidence suggest that immature oocytes are more vulnerable to the harmful effect of free radicals that can end by cell arrest [10].

Further more poor quality of retrieved oocytes could be attributed to accumulation of free radicals that result from exogenous gonadotropin adminstra- 
tion [11].

\section{Oxidative Stress and Endometriosis}

Endometriosis is a benign chronic gynecologic disorder characterized by dysmenorrhea starting before the menstrual period and continuing throughout the cycle until the end of the menstrual flow, dyspareunia, pelvic pain, and subfertility. It accounts for approximately $30 \%-50 \%$ of female infertility [12].

Endometriosis has long been known to be a disease of theories, however recent researches suggest that oxidative stress could be the key factor for the pathogenesis and progression of the disease.

For decades Sampson's theory linked retrograde menstruation to the development of endometriosis. Recent evidence suggests that peritoneal iron overload impairs the functionality of protective immune cells, rather than simply implantation of sheded endometrium [13].

This could be explained by the fact that once iron released from the haemoglobin it is considered as a toxic substance, that will trigger a series of reactions that ultimately generate free radicals which are toxic to the sperm and impair its motility, in addition can arrest embryo development, which explains the infertility in patients with early stage endometriosis.

Further more free radicals that are generated in endometriomas have toxic effect on the surrounding oocytes.

Recent Data explored the effectiveness of antioxidants on clinical improvement of dysmenorrhea and dyspaurnia.

\section{Oxidative Stress and Polycystic Ovary Syndrome}

Polycystic ovary syndrome (PCOS) is a heterogeneous endocrinological disease, affecting considerable number of women of childbearing age with a prevalence of $10 \%-15 \%$, characterized by chronic anovulation with oligoamenorrhea, obesity, enlarged cystic ovaries, hyperandrogenism and infertility.

Insulin resistance is encountered in approximately $50 \%-70 \%$ in patients with PCOS [14].

Recent researches suggest that deficiency of antioxidants is the responsible factor for reducing the sensitivity of insulin receptors. As consequences compensatory hyperinsulinemia resulted which augments luteinizing hormone, subsequently androgen production increased either via its own receptors or via insulin growth factor (IGF-1) receptors [15].

Acceleration of antral follicle atresia (Accelerated Apoptosis) is enhanced by excessive ROS which in turn will lead to the increase of androgens through the inability to be converted to estrogen.

In addition, oxygen radicals promote completion of the first meiotic division which explains the persistence of immature follicles in PCOS.

Still the question exists whether the abnormal levels of free radicals in PCOS derive from PCOS itself or whether it results from the associated complications. 


\section{Oxidative Stress and Reproductive Techniques}

Assisted Reproductive Techniques (ART) continues to be the hope for many couples where natural conception is not possible. With all the improvement in fertilization and implantation rate, ART will never mimic the natural pregnancy, due to lack of physiological defense mechanisms, in addition to existance of external sources of ROS.

Increasing evidence supports the imporatance of high concentration of total antioxidant capacity (TAC) for successful fertilization. On the other hand defciency can explain poor quality embryos.

Human gametes possess natural antioxidant defenses. A decrease in their total antioxidant capacity (TAC) may lead to oxidative stress. The levels of these antioxidants may be indicative of the extent of oxidative stress.

Preparation of semen can activate free radicals' production through centrifugation of immature spermatozoa. Furthermore, DNA damage secondary to oxidative stress could exist in the majotity of sperms selected in ART.

Interstingly oocytes and embryos are major sources of ROS as they use oxygen to produce energy through mitochondrial oxidative phosphorylation.

Continuous efforts have been made to mimic the invivo oxygen tension that embryos are exposed to, which ranges from $2 \%-8 \%$. Invitro exposure to higher oxygen concentrations has a harmful effect on late stage development of human embryos secondary to overproduction of free radicals and culturing blastocytes in low oxygen conditions has been shown to have a positive impact on live birth rate [16].

Exposure to direct light is another threat for the gametes and developing embryos, through damage to the unsaturated lipids in the cell membrane.

Studies have shown that ROS in conventional IVF (Invitro Fertilization) is more pronounced when compared to ICSI ( Intracytoplasmic sper Injecttion).In conventional IVF, oocytes may be exposed to high concentrations of spermatozoa for up to 20 hours, this prolonged co-incubation time increases exposure to ROS produced by spermatozoa.Reduced co-incubation time has been shown to increase embryo quality and clinical pregnancy rates.

This could be due to the presenc of cumulus cells in IVF, while its absent in ICSI as deuding the oocytes is essential step prior to incubation.

DNA fragmentation was higher in cryopreserved sperm when compared to fresh testicular one.

Oocyte cryopreservation-thawing process increase the susceptibility to ROSinduced damage.

Iincreased levels of 8-hydroxy-2-deoxyguanosine was found in poor quality embryos, which indicates DNA damage secondary to over production of free radicals.

Reported data showed that using melatonin as a drug to improve oocytes quality; was successful in achieving a high fertilization rate and reducing the number of degenerated follicles, by preventing DNA damage through its anti- 
oxidant action.

The solution for repeated implantation failure could be through optimising embryo quality as well as improving endometrial receptivity.

\section{Conclusions}

Sufficient evidence exists that oxidative stress can jeopardize fertilization, implantation and embryo viability.

Supplementation of antioxidants may have positive impact on female fertility.

Future studies should further explore the fundamental role of antioxidants for optimum reproductive function and ART outcome.

\section{References}

[1] Showell, M.G., Mackenzie-Proctor, R., Jordan, V. and Hart, R.J. (2017) Antioxidants for Female Subfertility. Cochrane Database of Systematic Reviews, No. 7.

[2] Pham-Huy, L.A., He, H. and Pham-Huy, C. (2008) Free Radicals, Antioxidants in Disease and Health. International Journal of Biomedical Science, 4, 89-96.

[3] Birben, E., Sahiner, U.M., Sackesen, C., Erzurum, S. and Kalayci, O. (2012) Oxidative Stress and Antioxidant Defense. World Allergy Organization Journal, 5, 9-19. https://doi.org/10.1097/WOX.0b013e3182439613

[4] Mylonas, C. and Kouretas, D. (1999) Lipid Peroxidation and Tissue Damage. In Vivo, 13, 295-309.

[5] Lobo, V., Patil, A. and Chandra, N. (2010) Free Radicals, Antioxidants and Functional Foods: Impact on Human Health. Pharmacognosy Reviews, 4, 118-126. https://doi.org/10.4103/0973-7847.70902

[6] Ruder, E.H., Hartman, T.J., Blumberg, J. and Goldman, M.B. (2008) Oxidative Stress and Antioxidants: Exposure and Impact on Female Fertility. Human Reproduction Update, 14, 345-357. https://doi.org/10.1093/humupd/dmn011

[7] Agarwal, A., Aponte-Mellado, A., Premkumar, B.J., Shaman, A. and Gupta, S. (2012) The Effects of Oxidative Stress on Female Reproduction: A Review. Reproductive Biology and Endocrinology, 10, 49. https://doi.org/10.1186/1477-7827-10-49

[8] Prasad, S., Tiwari, M., Pandey, A.N., Shrivastav, T.G. and Chaube, S.K. (2016) Impact of Stress on Oocyte Quality and Reproductive Outcome. Journal of Biomedical Science, 23, 36. https://doi.org/10.1186/s12929-016-0253-4

[9] Tripathi, A, Khatun, S., Pandey, A.N., Mishra, S.K., Chaube, R., Shrivastava, T.G., et al. (2009) Intracellular Levels of Hydrogen Peroxide and Nitric Oxide in Oocyte at Various Stages of Meiotic Cell Cycle and Apoptosis. Free Radical Research, 43, 287-294. https://doi.org/10.1080/10715760802695985

[10] Chaube, S.K., Prasad, P.V., Thakur, S.C. and Shrivastava, T.G. (2005) Hydrogen Peroxide Modulates Meiotic Cell Cycle and Induces Morphological Features Characteristics of Apoptosis in Rat Oocytes Cultured in Vitro. Apoptosis, 10, 863-875. https://doi.org/10.1007/s10495-005-0367-8

[11] Chao, H.T., Lee, S.Y., Lee, H.M., Liao, T.L., Wei, Y.H. and Kao, S.H. (2005) Repeated Ovarian Stimulations Induce Oxidative Damage and Mitochondrial DNA Mutations in Mouse Ovaries. Annals of the New York Academy of Sciences, 1042, 148-115. https://doi.org/10.1196/annals.1338.016 
[12] Donnez, J., Chantraine, F. and Nisolle, M. (2002) The Efficacy of Medical and Surgical Treatment of Endometriosis-Associated Infertility: Arguments in Favour of a Medico-Surgical Approach. Human Reproduction Update, 8, 89-94. https://doi.org/10.1093/humupd/8.1.89

[13] Yamaguchi, K., Mandai, M., Toyokuni, S., Hamanishi, J., Higuchi, T., Takakura, K. and Fujii, S. (2008) Contents of Endometriotic Cysts, Especially the High Concentration of Free Iron, Are a Possible Cause of Carcinogenesis in the Cysts through the Iron-Induced Persistent Oxidative Stress. Clinical Cancer Research, 14, 32-40. https://doi.org/10.1158/1078-0432.CCR-07-1614

[14] Legro, R.S., Castracane, V.D. and Kauffman, R.P. (2004) Detecting Insulin Resistance in Polycystic Ovary Syndrome: Purposes and Pitfalls. Obstetrical \& Gynecological Survey, 59, 141-154. https://doi.org/10.1097/01.OGX.0000109523.25076.E2

[15] Alchami, A., O’Donovan, O. and Davies, M. (2015) PCOS: Diagnosis and Management of Related Infertility. Obstetrics, Gynaecology \& Reproductive Medicine, 25, 279-282.

[16] Guo, N., Li, Y.F., Ai, J.H., Gu, L.J., Chen, W. and Liu, Q. (2014) Two Different Concentrations of Oxygen for Culturing Precompaction Stage Embryos on Human Embryo Development Competence: A Prospective Randomized Sibling-Oocyte Study. International Journal of Clinical and Experimental Pathology, 7, 6191-6198. 